\title{
Power on digraphs
}

Citation for published version (APA):

Peters, H., Timmer, J., \& van den Brink, R. (2016). Power on digraphs. Maastricht University, Graduate School of Business and Economics. GSBE Research Memoranda No. 019 https://doi.org/10.26481/umagsb.2016019

Document status and date:

Published: 01/04/2016

DOI:

10.26481/umagsb.2016019

Document Version:

Publisher's PDF, also known as Version of record

\section{Please check the document version of this publication:}

- A submitted manuscript is the version of the article upon submission and before peer-review. There can be important differences between the submitted version and the official published version of record.

People interested in the research are advised to contact the author for the final version of the publication, or visit the DOI to the publisher's website.

- The final author version and the galley proof are versions of the publication after peer review.

- The final published version features the final layout of the paper including the volume, issue and page numbers.

Link to publication

\footnotetext{
General rights rights.

- You may freely distribute the URL identifying the publication in the public portal. please follow below link for the End User Agreement:

www.umlib.nl/taverne-license

Take down policy

If you believe that this document breaches copyright please contact us at:

repository@maastrichtuniversity.nl

providing details and we will investigate your claim.
}

Copyright and moral rights for the publications made accessible in the public portal are retained by the authors and/or other copyright owners and it is a condition of accessing publications that users recognise and abide by the legal requirements associated with these

- Users may download and print one copy of any publication from the public portal for the purpose of private study or research.

- You may not further distribute the material or use it for any profit-making activity or commercial gain

If the publication is distributed under the terms of Article $25 \mathrm{fa}$ of the Dutch Copyright Act, indicated by the "Taverne" license above, 


\section{Maastricht University}

Hans Peters, Judith Timmer, Rene van den Brink

\section{Power on digraphs}

RM/16/019

\section{GSBE}

Maastricht University School of Business and Economics

Graduate School of Business and Economics

P.O Box 616

NL-6200 MD Maastricht

The Netherlands 


\title{
Power on digraphs
}

\author{
Hans Peters* Judith Timmer ${ }^{\dagger} \quad$ Rene van den Brink ${ }^{\ddagger}$
}

This version, April 2016

\begin{abstract}
It is assumed that relations between $n$ players are reflected by a directed graph or digraph. Such a digraph is called invariant if there is an arc between any two players between whom there is also a directed path. We characterize a class of power indices for invariant digraphs based on four axioms: Null Player, Constant Sum, Anonymity, and the Transfer Property. This class is determined by $2 n-2$ parameters. By considering additional conditions about the effect of adding a directed link (arc) between two players we single out three different, one parameter families of power indices, reflecting several well-known indices from the literature: Copeland score, $\beta$ - and apex-type indices.
\end{abstract}

\section{JEL-Classification numbers C71}

Keywords Digraph, power index, transfer property, link addition

\section{Introduction}

We consider situations where the relations between $n$ players are reflected by a directed graph or digraph. There are several interpretations possible. A directed link (or arc) from player $i$ to player $j$ may reflect that player $i$ controls player $j$, for instance $i$ is an investor who has the majority of the shares of firm $j$. With this interpretation our paper is related to Gambarelli and Owen (1994), where the players are firms or investment companies. It is also a special case of the approach by $\mathrm{Hu}$ and Shapley $(2003 \mathrm{a}, \mathrm{b})$ and of the mutual control structures of Karos and Peters (2015). Somewhat related, our model can also be seen as a permission structure as introduced in Gilles et al (1992). A directed link from player $i$ to player $j$ then reflects the fact that player $j$ needs the permission of player $i$ in order to cooperate with other players. Still another interpretation is an information structure: player $j$ can get information from player $i$ only if

\footnotetext{
*Department of Quantitative Economics, Maastricht University, The Netherlands. Email: h.peters@maastrichtuniversity.nl.

†Stochastic Operations Research, Department of Applied Mathematics, University of Twente, P.O. Box 217, 7500 AE Enschede, The Netherlands. Email: j.b.timmer@utwente.nl.

${ }^{\ddagger}$ Department of Econometrics, Free University of Amsterdam, Email: j.r.vanden.brink@vu.nl.
} 
there is a directed link from player $i$ to player $j$. These are applications where the links are between decision making agents. Other types of applications have the arcs representing the results of matches played among teams in a sports competition, or the structure of links between internet pages.

A digraph will be called invariant if for any two players who are connected via a directed path, there is also a direct link. ${ }^{1}$ For instance, in the control interpretation, if player $i$ controls player $j$ and $j$ controls $k$ then player $i$ controls $k$ indirectly; in an invariant digraph there will be a direct link between $i$ and $k$. An invariant digraph is a special case of an invariant mutual control structure studied in Karos and Peters (2015).

Our aim in this paper is to study power indices, reflecting the power of players as a consequence of their positions in the digraph. Following Karos and Peters (2015), we impose four axioms on a power index: Null Player, Constant Sum, Anonymity, and the Transfer Property. The Transfer Property (first introduced in a different format by Dubey, 1975) is an additivity condition in the spirit of Shapley (1953). It is the main tool to ensure that a power index is completely determined by its values on digraphs of an elementary form. In these elementary digraphs there is a set $M$ of players who each have a link to one and the same player $j$. Due to the Anonymity condition, only the cardinality of $M$ matters, but it makes a difference whether or not player $j$ is in $M$ : this is why we arrive at a class of power indices with $2(n-1)$ degrees of freedom (parameters). The Null Player axiom ensures that players outside $M$ (other than possibly $j$ ) have zero power. Combined with the Constant Sum axiom we obtain that the sum of the player powers is always 0 . This is in contrast with usual power indices (e.g., Shapley and Shubik, 1954; see also Gambarelli, 1994, and Gambarelli and Stach, 2009), where power is between 0 and 1, with total 1. In our approach it is natural to allow for negative power. A null player is a player who has neither incoming nor outgoing links in the digraph, and it is natural to assign power 0 to such a player. In turn then, it is equally natural to allow for negative power for a player who has only incoming links. Our main result here (Theorem 4.4) is related to the main result in Karos and Peters (2015), but it nevertheless requires a new and different, though less involved, proof.

The Constant Sum axiom and the possibility of negative power are two features by which the indices in this paper distinguish themselves from digraph power measures such as the outdegree and $\beta$-measure, axiomatized in van den Brink and Gilles (2000), which both satisfy a normalization that implies that the sum of powers of the players depends on the digraph. We find three subclasses, related to the Copeland score, $\beta$-measure and apex-measure, by imposing three different additional conditions with respect to link addition. ${ }^{2}$ First, we impose

\footnotetext{
${ }^{1}$ Such digraphs are often called transitive. For more general mutual control structures as in Karos and Peters (2015), invariance is stronger than transitivity.

${ }^{2}$ The effect of link addition was introduced in communication graph games by Myerson (1977), who introduced the axiom of fairness, stating that deleting an undirected communication link between two players has the same effect on their payoffs. Together with so-called component efficiency this characterizes a Shapley type solution, later referred to as the Myerson value.
} 
the condition that adding a link from a player $i$ to a player $j$ does not change the power of the players who already had a link to player $j$. This may make sense, for instance, in a situation where player $j$ has to do tasks for players who have a link to $j$ - assuming there is no capacity constraint on what player $j$ can do. Imposing this condition (called Link Addition 1) on top of the four basic conditions above singles out a one-parameter family of power indices closely related to the Copeland score (Copeland, 1951) from social choice theory: the power of a player is proportional to the number of players to who he has a link minus the number of players who have a link to him.

The next condition requires that adding a link does not change the power of a player $j$ to whom this link is incoming. This will imply that now the players that already had a link to $j$ have to share the power they had with the newcomer. Also this condition (Link Addition 2) singles out a one-parameter family of power indices, this time closely related to the concept of a $\beta$-measure as in van den Brink and Gilles (2000) and van den Brink and Borm (2002).

The final condition that we consider (Link Addition 3) requires that adding a link from player $i$ to player $j$ equally increases or reduces the power of $i$ and $j$. Again, we obtain a one-parameter family of power indices, with the property that incoming links have no effect on a player's power. These power indices are similar to the apex-type power index in van den Brink (2002).

The organization of the paper is as follows. Section 2 introduces invariant digraphs, Section 3 the main axioms for a power index, Section 4 the main characterization result, and Section 5 the refinements based on Link Addition axioms. Section 6 concludes.

\section{Preliminaries}

For a set $A$ we denote by $P(A)$ the set of all subsets of $A$, and by $P_{0}(A)$ the set of all nonempty subsets of $A$. By $|A|$ we denote the number of elements of $A$.

Let $N=\{1, \ldots, n\}$ with $n \geq 2$ denote the set of players. Elements of $P(N)$ are called coalitions. A directed graph or digraph on $N$ is a map $C: P(N) \rightarrow$ $P(N)$ satisfying $C(\emptyset)=\emptyset$ and $C(S)=\cup_{i \in S} C(i)$ for every $S \in P_{0}(N) .{ }^{3}$ Hence, a digraph $C$ is completely determined by its values $C(i)$ for $i \in N$. The graphical interpretation of a digraph $C$ is, indeed, that there is an $\operatorname{arc}$ from $i \in N$ to $j \in N$ if and only if $j \in C(i) .{ }^{4}$ As mentioned in the Introduction, various interpretations with respect to applications are possible. The set $C(i)$ can be interpreted as the set of players controlled by player $i$ (cf. Hu and Shapley, 2003a,b; Karos and Peters, 2015), or the set of players who need permission from $i$ (Gilles et al, 1992); or the set of players to whom player $i$ can communicate, etc. The reason for defining a digraph not just for singletons lies in the axiomatic approach to power indices later on. Observe that a digraph $C$ is trivially monotonic: if

\footnotetext{
${ }^{3}$ In this way, digraphs can be seen as a special case of mutual control structures, see Karos and Peters (2015).

${ }^{4}$ Usually, what we call players are called nodes or vertices in a digraph. Because of the applications we have in mind, we refer to them as players.
} 
$S, T \in P(N)$ and $S \subseteq T$ then $C(S) \subseteq C(T)$.

For a digraph $C$, a directed path from $i \in N$ to $j \in N$ is a sequence $\left(i_{1}, i_{2}\right),\left(i_{2}, i_{3}\right), \ldots,\left(i_{k}, i_{k+1}\right) \in N \times N$ for some $k \in \mathbb{N}$ such that $i_{\ell+1} \in C\left(i_{\ell}\right)$ for each $\ell=1, \ldots, k, i=i_{1}$, and $j=i_{k+1}$. A digraph $C$ is invariant if for all $i, j \in N$, if there is a directed path from $i$ to $j$, then $j \in C(i) .{ }^{5}$ The expression 'invariant' refers to the fact that in an invariant digraph adding a link between players having a directed path connecting them, does not change the digraph. For instance, in the interpretation of a digraph as a mutual control structure as in Karos and Peters (2015), invariance means that all 'indirect' control relations are already included as direct controls.

Note that an arbitrary digraph can be made invariant by simply adding arcs for every pair of players between whom there exists a directed path. ${ }^{6}$ The following observation, the easy proof of which is omitted, will be convenient in the sequel.

Lemma 2.1. Let $C$ be a digraph. Then $C$ is invariant if and only if $C(i \cup$ $C(i))=C(i)$ for all $i \in N$.

We denote the set of all digraphs (on $N$ ) by $\mathcal{D}$ and the set of all invariant digraphs by $\mathcal{D}^{*}$.

In the sequel the following observations will be relevant. For $C, D \in \mathcal{D}$ define $C \cup D$ and $C \cap D$ by $(C \cup D)(S)=C(S) \cup D(S)$ and $(C \cap D)(S)=C(S) \cap D(S)$ for all $S \in P(N)$. Then $C \cup D \in \mathcal{D}$ but even if $C, D \in \mathcal{D}^{*}$ then not necessarily $C \cup D \in \mathcal{D}^{*}$. See the following example.

Example 2.2. Let $N=\{1,2,3\}$ and let $C, D \in \mathcal{D}^{*}$ be defined by $C(1)=$ $2, D(2)=3$, and $C(i)=\emptyset$ and $D(i)=\emptyset$ in the remaining cases. ${ }^{7}$ Then $(C \cup D)(1)=2$ and $(C \cup D)(2)=3$ but $3 \notin(C \cup D)(1)$, so that $C \cup D \notin \mathcal{D}^{*}$. (See also Remark 2.9 in Karos and Peters, 2015.)

Further, if $C, D \in \mathcal{D}^{*}$ then not necessarily $C \cap D \in \mathcal{D}$, but if in that case $C \cap D \in \mathcal{D}$ then also $C \cap D \in \mathcal{D}^{*}$. The latter follows as in Karos and Peters (2015), and is also straightforward to prove by using Lemma 2.1; for the former see the following example.

Example 2.3. Let $n \geq 4$ and let $C(1)=3, C(2)=4, D(1)=4, D(2)=3$, and $C(i)=D(i)=\emptyset$ for all $i \neq 1,2$. Then $C, D \in \mathcal{D}^{*}$ but $C \cap D \notin \mathcal{D}$ since $(C \cap D)(i)=\emptyset$ for all $i$ but $(C \cap D)(12)=34$.

Example 2.3 in fact shows that our definition of the intersection $C \cap D$ of two digraphs cannot be restricted to taking intersections for singleton coalitions only and then extend to arbitrary coalitions by taking unions.

\footnotetext{
${ }^{5}$ These digraphs are often refered to as transitive digraphs.

${ }^{6}$ See Karos and Peters, 2015, or Hu and Shapley, 2003 a,b, for procedures to make more general control structures invariant.

${ }^{7}$ Here and elsewhere we often omit set braces if confusion is unlikely.
} 


\section{Axioms for a power index}

We consider power indices for invariant digraphs. A power index is a map $\varphi: \mathcal{D}^{*} \rightarrow \mathbb{R}^{N}$. We propose four basic axioms, in the spirit of the standard axioms of the Shapley (1953) value for cooperative games. As in Karos and Peters (2015) we will then prove that these four axioms characterize a large, $(2 n-2)$-parameter family of power indices.

We say that $i \in N$ is a null player in $C \in \mathcal{D}^{*}$ if $C(i)=\emptyset$ and $i \notin C(N)$ : player $i$ is an isolated node in the digraph. The digraph in which every player is a null player, is denoted by $O$, i.e., $O(S)=\emptyset$ for all $S \subseteq N$. This corresponds to a graph without any arcs.

Let $\pi: N \rightarrow N$ be a permutation. Then for $C \in \mathcal{D}^{*}$ we define $\pi C \in \mathcal{D}^{*}$ by

$$
(\pi C)(S)=\pi\left(C\left(\pi^{-1}(S)\right)\right) .
$$

The first axiom requires that null players have zero power.

Null Player (NP) $\varphi_{i}(C)=0$ for every null player $i$ in $C$, for every $C \in \mathcal{D}^{*}$.

The second axiom states that the sum of powers of all players is the same for any digraph.

Constant Sum (CS) $\sum_{i \in N} \varphi_{i}(C)=\sum_{i \in N} \varphi_{i}(D)$ for all $C, D \in \mathcal{D}^{*}$.

The combination of these two axioms has as a simple consequence that the powers of all players in a digraph always add up to zero.

Lemma 3.1. Let $\varphi$ be a power index satisfying NP and CS. Then $\sum_{i \in N} \varphi_{i}(C)=$ 0 for every $C \in \mathcal{D}^{*}$.

Proof. By NP, $\varphi_{i}(O)=0$ for every $i \in N$. Hence, by CS, $\sum_{i \in N} \varphi_{i}(C)=$ $\sum_{i \in N} \varphi_{i}(O)=0$ for every $C \in \mathcal{D}^{*}$.

Usually in the literature, power indices take values between 0 and 1 and sum up to 1 (e.g., Shapley and Shubik, 1954). In our case, we allow for the fact that power can be negative. Typically, for instance, a null player may be regarded to be more powerful than a player $j$ with $C(j)=\emptyset$ but $j \in C(i)$ for some other player $i$, so that we may wish to assign negative power to such a player $j$.

The next axiom is standard.

Anonymity (AN) $\varphi_{\pi(i)}(\pi C)=\varphi_{i}(C)$ for every player $i \in N$, every permutation $\pi$ of $N$, and every $C \in \mathcal{D}^{*}$.

The final axiom replaces the usual additivity condition known from the Shapley value. For cooperative games it was first introduced by Dubey (1975) in the format (1) in the next section. See, further, Dubey et al (2005) and Einy and Haimanko (2011). 
Transfer Property (TP) $\varphi(C)-\varphi\left(C^{\prime}\right)=\varphi(D)-\varphi\left(D^{\prime}\right)$ for all $C, C^{\prime}, D, D^{\prime} \in$ $\mathcal{D}^{*}$ such that $C^{\prime} \subseteq C, D^{\prime} \subseteq D$, and $C(S) \backslash C^{\prime}(S)=D(S) \backslash D^{\prime}(S)$ for every $S \subseteq N$.

An alternative and stronger version of the Transfer Property would be obtained by imposing the condition $C(S) \backslash C^{\prime}(S)=D(S) \backslash D^{\prime}(S)$ only for singletons, i.e., replacing it by $C(i) \backslash C^{\prime}(i)=D(i) \backslash D^{\prime}(i)$ for every $i \in N$. This, however, is too strong for our purposes: it would have the same effect as adding the Link Addition 1 condition, see Section 5, and excluding other power indices, for instance those characterized in that section.

\section{Characterization of power indices satisfying NP, CS, AN, and TP}

The main result of this section is Theorem 4.4, which is a characterization of all power indices satisfying Null Player, Constant Sum, Anonymity, and the Transfer Property. We start out with proving a consequence of the Transfer Property.

Lemma 4.1. Let $\varphi$ be a power index satisfying TP. Then

$$
\varphi(C \cap D)+\varphi(C \cup D)=\varphi(C)+\varphi(D)
$$

for all $C, D \in \mathcal{D}^{*}$ with $C \cup D, C \cap D \in \mathcal{D}^{*}$.

Proof. Let $C, D \in \mathcal{D}^{*}$ with $C \cup D, C \cap D \in \mathcal{D}^{*}$. Clearly,

$$
(C(S) \cup D(S)) \backslash C(S)=D(S) \backslash(C(S) \cap D(S))
$$

for all $S \subseteq N$. Hence by TP, $\varphi(C \cup D)-\varphi(C)=\varphi(D)-\varphi(C \cap D)$, implying (1).

For $C \in \mathcal{D}^{*}$ and $j \in N$ we define the digraph $C_{j}$ by

$$
C_{j}(i)=\left\{\begin{array}{cl}
\{j\} & \text { if } j \in C(i) \\
\emptyset & \text { otherwise. }
\end{array}\right.
$$

It is easy to see that $C_{j} \in \mathcal{D}^{*}$. Observe that in a graphical representation of $C_{j}$, the graph has only arcs pointing to $j$, namely from those players $i$ with $j \in C(i)$.

Lemma 4.2. Let $\varphi$ be a power index satisfying $T P$ and $N P$, and let $C \in \mathcal{D}^{*}$. Then $\varphi(C)=\sum_{j \in N} \varphi\left(C_{j}\right)$.

Proof. We first show that $\cup_{k \in T} C_{k} \in \mathcal{D}^{*}$ for every $T \in P_{0}(N)$. Let $S, T \in P_{0}(N)$, then

$$
\bigcup_{k \in T} C_{k}(S)=\bigcup_{k \in T} \bigcup_{j \in S} C_{k}(j)=\bigcup \bigcup_{j \in S} C_{k \in T}(j)
$$


which implies that $\cup_{k \in T} C_{k} \in \mathcal{D}$. Let $i \in N$ and $j \in \cup_{k \in T} C_{k}\left(i \cup\left(\cup_{h \in T} C_{h}(i)\right)\right)$, then there is $\ell \in T$ such that $j \in C_{\ell}\left(i \cup\left(\cup_{h \in T} C_{h}(i)\right)\right)$. In turn, since $C_{\ell} \in \mathcal{D}$, this implies that $j \in C_{\ell}(i)$ or there is $m \in T$ such that $j \in C_{m}(i)$. Hence, $j \in$ $\cup_{k \in T} C_{k}(i)$, so $\cup_{k \in T} C_{k}\left(i \cup\left(\cup_{h \in T} C_{h}(i)\right)\right) \subseteq \cup_{k \in T} C_{k}(i)$. The converse inclusion follows by monotonicity, hence $\cup_{k \in T} C_{k} \in \mathcal{D}^{*}$ by Lemma 2.1 .

Next, for every $T \in P_{0}(N)$ and $k \notin T$, we have $C_{k} \cap\left(\cup_{\ell \in T} C_{\ell}\right)=O \in \mathcal{D}^{*}$ (the empty digraph). By NP, $\varphi(O)=0 \in \mathbb{R}^{n}$. Also, $C=\cup_{i \in N} C_{i}$.

By the preceding arguments and by repeatedly applying (1), we obtain

$$
\begin{aligned}
\varphi(C) & =\varphi\left(C_{1} \cup\left(\cup_{i=2, \ldots, n} C_{i}\right)\right) \\
& =\varphi\left(C_{1}\right)+\varphi\left(\cup_{i=2, \ldots, n} C_{i}\right)-\varphi\left(C_{1} \cap\left(\cup_{i=2, \ldots, n} C_{i}\right)\right) \\
& =\varphi\left(C_{1}\right)+\varphi\left(\cup_{i=2, \ldots, n} C_{i}\right) \\
& \vdots \\
& =\sum_{j \in N} \varphi\left(C_{j}\right),
\end{aligned}
$$

which concludes the proof of the lemma.

By Lemma 4.2 we may concentrate on digraphs of the form $C_{j}$. More generally, for $M \in P_{0}(N)$ and $j \in N$ the digraph $U_{M, j}$ is defined by

$$
U_{M, j}(i)=\left\{\begin{array}{cl}
\{j\} & \text { if } i \in M \\
\emptyset & \text { otherwise. }
\end{array}\right.
$$

Clearly, $U_{M, j}$ is invariant.

Lemma 4.3. Let the power index $\varphi$ on $\mathcal{D}^{*}$ satisfy $N P, C S$, and $A N$. Then there are $\alpha_{0}, \alpha_{1}, \ldots, \alpha_{n-1}, \beta_{1}, \ldots, \beta_{n} \in \mathbb{R}$, with $\alpha_{0}=0, \beta_{1}=0$, such that for every $M \in P(N)$ and $j \in N$, with $m=|M|$ :

(a) if $j \notin M$ then for every $i \in N$

$$
\varphi_{i}\left(U_{M, j}\right)=\left\{\begin{array}{cl}
0 & \text { if } i \notin M \cup j \\
\alpha_{m} / m & \text { if } i \in M \\
-\alpha_{m} & \text { if } i=j
\end{array}\right.
$$

(b) if $j \in M$ then for every $i \in N$

$$
\varphi_{i}\left(U_{M, j}\right)=\left\{\begin{array}{cl}
0 & \text { if } i \notin M \\
\beta_{m} / m & \text { if } i \in M \backslash j \\
\beta_{m} / m-\beta_{m} & \text { if } i=j
\end{array}\right.
$$

Proof. Straightforward from the axioms.

Observe that for $C \in \mathcal{D}^{*}$ and $j \in N$, we have $C_{j}=U_{M_{j}^{C}, j}$ where $M_{j}^{C}=\{i \in$ $N: j \in C(i)\}$.

From Lemmas 4.2 and 4.3 we obtain our main result. 
Theorem 4.4. A power index $\varphi$ on $\mathcal{D}^{*}$ satisfies NP, CS, AN, and TP if and only if there are $\alpha_{0}, \alpha_{1}, \ldots, \alpha_{n-1}, \beta_{1}, \ldots, \beta_{n} \in \mathbb{R}$, with $\alpha_{0}=0$ and $\beta_{1}=0$, such that for each $C \in \mathcal{D}^{*}$ we have $\varphi(C)=\sum_{j \in N} \varphi\left(C_{j}\right)$, with each $\varphi\left(C_{j}\right)=$ $\varphi\left(U_{M_{j}^{C}, j}\right)$ defined as in (a) and (b) of Lemma 4.3.

Proof. The only-if direction follows from Lemmas 4.2 and 4.3. For the ifdirection, let $\alpha_{0}, \alpha_{1}, \ldots, \alpha_{n-1}, \beta_{1}, \ldots, \beta_{n} \in \mathbb{R}$, with $\alpha_{0}=0$ and $\beta_{1}=0$, and with $\varphi(C)=\sum_{j \in N} \varphi\left(C_{j}\right)$ with each $\varphi\left(C_{j}\right)=\varphi\left(U_{M_{j}^{C}, j}\right)$ defined as in (a) and (b) of Lemma 4.2. AN and CS of $\varphi$ are obvious. For NP, note that $i$ is a null player in $C \in \mathcal{D}^{*}$ if and only if $C_{i}=O$ and $i \notin M_{j}^{C}$ for all $j \in N$. This implies that $\varphi_{i}\left(C_{j}\right)=0$ for all $j \in N$ and therefore $\varphi_{i}(C)=0$.

For TP, let $C^{\prime}, C, D^{\prime}, D \in \mathcal{D}^{*}$ such that $C^{\prime} \subseteq C, D^{\prime} \subseteq D$, and $C(S) \backslash C^{\prime}(S)=$ $D(S) \backslash D^{\prime}(S)$ for every $S \subseteq N$. Then it is straightforward to show that $C_{j}^{\prime} \subseteq C_{j}$, $D_{j}^{\prime} \subseteq D_{j}$, and $C_{j} \backslash C_{j}^{\prime}=D_{j} \backslash D_{j}^{\prime}$ for all $j \in N$. Hence, to show TP, it is sufficient to show that

$$
\varphi\left(C_{j}\right)-\varphi\left(C_{j}^{\prime}\right)=\varphi\left(D_{j}\right)-\varphi\left(D_{j}^{\prime}\right)
$$

for all $j \in N$. Now fix $j$ and observe that $C_{j}^{\prime} \subseteq C_{j}$ and $D_{j}^{\prime} \subseteq D_{j}$ imply $M_{j}^{C^{\prime}} \subseteq M_{j}^{C}$ and $M_{j}^{D^{\prime}} \subseteq M_{j}^{D}$. If $M_{j}^{C^{\prime}}=M_{j}^{C}$, then $C_{j}^{\prime}=C_{j}$ and therefore $D_{j}^{\prime}=D_{j}$, so that $(*)$ follows. Otherwise, both $M_{j}^{C^{\prime}} \varsubsetneqq M_{j}^{C}$ and $M_{j}^{D^{\prime}} \varsubsetneqq M_{j}^{D}$. Then, for $S=N \backslash M_{j}^{C^{\prime}}$ we have $C_{j}(S) \backslash C_{j}^{\prime}(S)=\{j\}=D_{j}(S) \backslash D_{j}^{\prime}(S)$. This implies $S \cap M_{j}^{D^{\prime}}=\emptyset$, hence $M_{j}^{D^{\prime}} \subseteq M_{j}^{C^{\prime}}$. Similarly, $M_{j}^{C^{\prime}} \subseteq M_{j}^{D^{\prime}}$, hence $M_{j}^{D^{\prime}}=M_{j}^{C^{\prime}}$. This implies that also $M_{j}^{D}=M_{j}^{C}$, so $C_{j}=D_{j}$ and $C_{j}^{\prime}=D_{j}^{\prime}$, and $(*)$ follows again.

In the concluding Section 6 we provide examples to show that the four axioms in Theorem 4.4 are logically independent.

\section{Adding links: Copeland, $\beta$ - and apex-type in- dices}

In this section we consider the class of power indices characterized in Theorem 4.4 and study refinements following from effects of adding a directed link ( $\operatorname{arc})$ between a player $i$ and a player $j$. Let $\Phi$ denote the class of all power indices satisfying NP, CS, AN, and TP. By Theorem 4.4, a generic element of $\Phi$ can be denoted as $\varphi^{\alpha, \beta}$, where $\alpha=\left(\alpha_{1}, \ldots, \alpha_{n-1}\right) \in \mathbb{R}^{n-1}$ and $\beta=\left(\beta_{2}, \ldots, \beta_{n}\right) \in \mathbb{R}^{n-1}$.

The first axiom says that if we add an additional link to a player $j$ from some player $i$ then this should not change the power of the players who already have a link to $j$. In the control or permission parlance: if player $j$ gets additionally controlled by some player $i$ then this should not change the power of the players who were already controlling $j$.

Recall that for a digraph $C \in \mathcal{D}^{*}$ and a player $j, M_{j}^{C}$ is the set of players with a link to $j$, i.e., $M_{j}^{C}=\{i \in N: j \in C(i)\}$. 
Link Addition 1 (LA1) $\varphi_{h}(C)=\varphi_{h}\left(C^{\prime}\right)$ for all $C, C^{\prime} \in \mathcal{D}^{*}, j \in N$, and $h \in M_{j}^{C} \backslash\{j\}$, such that there is $i \in N$ with $i \notin M_{j}^{C}, C^{\prime}(i)=C(i) \cup\{j\}$ and $C^{\prime}(\ell)=C(\ell)$ for all $\ell \in N \backslash\{i\}$.

Theorem 5.1. Let $\varphi=\varphi^{\alpha, \beta} \in \Phi$. Then $\varphi$ satisfies LA1 if and only if there is a $c \in \mathbb{R}$ such that $\alpha_{k}=k c$ for all $k=1, \ldots, n-1$ and $\beta_{k}=k c$ for all $k=2, \ldots, n$.

Thus, under LA1 we obtain a one-parameter family of power indices of the form

$$
\varphi_{i}^{c}(C)=\left(\sum_{j \in C(i)} c\right)-\left|M_{i}^{C}\right| c=c\left(|C(i)|-\left|M_{i}^{C}\right|\right),
$$

where $c \in \mathbb{R}$. For instance, for $c=1$ and in the control terminology, the power of player $i$ is equal to the number of players controlled by player $i$ minus the number of players controlling player $i$, which is similar to the Copeland score in social choice theory (Copeland, 1951). ${ }^{8}$

Proof of Theorem 5.1 For the if-direction let $c \in \mathbb{R}$ and let $\varphi^{c}$ be as in (3). We show that $\varphi^{c}$ satisfies LA1. Let $C, C^{\prime}, h, j, i$ be as in the statement of the axiom. Then $h \neq i$ and therefore $\varphi_{h}^{c}(C)=c\left(|C(h)|-\left|M_{h}^{C}\right|\right)=c\left(\left|C^{\prime}(h)\right|-\left|M_{h}^{C^{\prime}}\right|\right)=$ $\varphi_{h}^{c}\left(C^{\prime}\right)$.

For the only-if direction, let $\varphi=\varphi^{\alpha, \beta} \in \Phi$ satisfy LA1. First suppose $M \in P_{0}(N)$ and $i, j \in N \backslash M$ with $i \neq j$ and $|M|=k$ for some $k \in\{1, \ldots, n-2\}$. Then for $h \in M$, by LA1 with $U_{M, j}$ in the role of $C$ and $U_{M \cup\{i\}, j}$ in the role of $C^{\prime}, \varphi_{h}\left(U_{M, j}\right)=\varphi_{h}\left(U_{M \cup\{i\}, j}\right)$, hence $\alpha_{k} / k=\alpha_{k+1} /(k+1)$. Let $c=\alpha_{1}$, then this implies that $\alpha_{k}=k c$ for all $k=1, \ldots, n-1$. Now suppose $M^{\prime} \in P_{0}(N)$ with $j \notin M^{\prime}$ and $\left|M^{\prime}\right|=k \in\{1, \ldots, n-1\}$. For $h \in M^{\prime}$, again by LA1, $\varphi_{h}\left(U_{M^{\prime}, j}\right)=\varphi_{h}\left(U_{M^{\prime} \cup\{j\}, j}\right)$, hence $\alpha_{k} / k=\beta_{k+1} /(k+1)$. This implies $\beta_{k}=k c$ for all $k=2, \ldots, n-1$.

The following axiom requires that it is player $j$ whose power does not change if an additional link is added from some player $i$ to $j$. In terms of control: if a player $j$ becomes additionally controlled by some player $i$ then this should not change the power of player $j$.

Link Addition 2 (LA2) $\varphi_{j}(C)=\varphi_{j}\left(C^{\prime}\right)$ for all $C, C^{\prime} \in \mathcal{D}^{*}, j \in N$ with $M_{j}^{C} \backslash\{j\} \neq \emptyset$, and $i \in N$ with $i \notin M_{j}^{C}, C^{\prime}(i)=C(i) \cup\{j\}$ and $C^{\prime}(\ell)=C(\ell)$ for all $\ell \in N \backslash\{i\}$.

Theorem 5.2. Let $\varphi=\varphi^{\alpha, \beta} \in \Phi$. Then $\varphi$ satisfies LA2 if and only if there is a $c \in \mathbb{R}$ such that $\alpha_{k}=c$ for all $k=1, \ldots, n-1$ and $\beta_{k}=\frac{k}{k-1} c$ for all $k=2, \ldots, n$.

The power indices characterized in Theorem 5.2 take the form

$$
\bar{\varphi}_{i}^{c}(C)=\sum_{j \in C(i) \backslash\{i\}} \frac{c}{\left|M_{j}^{C} \backslash\{j\}\right|}-c 1_{\left\{M_{i}^{C} \neq \emptyset\right\}}
$$

\footnotetext{
${ }^{8}$ An alternative way of characterizing the class $\left\{\varphi^{C}: c \in \mathbb{R}\right\}$ is by strengthening the Transfer Property in the way indicated at the end of Section 3.
} 
where $1_{\{P\}}=1$ if statement $P$ is true and $1_{\{P\}}=0$ otherwise. A power index $\bar{\varphi}^{c}$ is similar to the idea of the $\beta$-measure as in van den Brink and Gilles (2000) or its reflexive variant in van den Brink and Borm (2002): if a player $i$ has a link to a player $j$, then he equally shares the amount of power $c$ with the other players having a link to $j$, except possibly $j$. The difference is that player $j$ loses $c$ in power.

Proof of Theorem 5.2 For the if-direction let $c \in \mathbb{R}$ and $\bar{\varphi}^{c}$ as in (4). We show that $\bar{\varphi}^{c}$ satisfies LA2. Let $C, C^{\prime}, j, i$ be as in the statement of the axiom, then

$$
\begin{aligned}
\bar{\varphi}_{j}^{c}(C) & =\sum_{\ell \in C(j) \backslash\{j\}} \frac{c}{\left|M_{\ell}^{C} \backslash\{\ell\}\right|}-c \\
& =\sum_{\ell \in C^{\prime}(j) \backslash\{j\}} \frac{c}{\left|M_{\ell}^{C^{\prime}} \backslash\{\ell\}\right|}-c \\
& =\bar{\varphi}_{j}^{c}\left(C^{\prime}\right)
\end{aligned}
$$

as is straightforward to verify, both for the case $i \neq j$ and for the case $i=j$.

For the only-if direction, let $\varphi=\varphi^{\alpha, \beta} \in \Phi$ satisfy LA2. First suppose $M \in P_{0}(N)$ and $i, j \in N \backslash M$ with $i \neq j$ and $|M|=k$ for some $k \in\{1, \ldots, n-$ $2\}$. Then by LA2, with $U_{M, j}$ in the role of $C$ and $U_{M \cup\{i\}, j}$ in the role of $C^{\prime}$, $\varphi_{j}\left(U_{M, j}\right)=\varphi_{j}\left(U_{M \cup\{i\}, j}\right)$, hence $\alpha_{k}=\alpha_{k+1}$. Let $c=\alpha_{1}$, then this implies that $\alpha_{k}=c$ for all $k=1, \ldots, n-1$. Now suppose $M^{\prime} \in P_{0}(N)$ with $j \notin M^{\prime}$ and $\left|M^{\prime}\right|=k \in\{1, \ldots, n-1\}$. Again by LA2, $\varphi_{j}\left(U_{M^{\prime}, j}\right)=\varphi_{j}\left(U_{M^{\prime} \cup\{j\}, j}\right)$, hence by the previous argument, $c=\frac{\beta_{k+1}}{k+1}-\beta_{k+1}=\frac{-k}{k+1} \beta_{k+1}$, and thus $\beta_{k+1}=\frac{k+1}{k} c$, which implies that $\beta_{k}=\frac{k}{k-1} c$ for all $k=2, \ldots, n-1$.

The final axiom we consider says that if we add a link from a player $i$ to a player $j$ then both have the same gain or loss in power.

Link Addition 3 (LA3) $\varphi_{i}\left(C^{\prime}\right)-\varphi_{i}(C)=\varphi_{j}\left(C^{\prime}\right)-\varphi_{j}(C)$ for all $C, C^{\prime} \in \mathcal{D}^{*}$, $j \in N$ with $M_{j}^{C} \neq \emptyset$, and $i \in N$ with $i \notin M_{j}^{C}, C^{\prime}(i)=C(i) \cup\{j\}$ and $C^{\prime}(\ell)=C(\ell)$ for all $\ell \in N \backslash\{i\}$.

Theorem 5.3. Let $\varphi=\varphi^{\alpha, \beta} \in \Phi$. Then $\varphi$ satisfies LA3 if and only if there is a $c \in \mathbb{R}$ such that $\alpha_{k}=\frac{2}{k+1}$ c for all $k=1, \ldots, n-1$, and $\beta_{k}=0$ for all $k=2, \ldots, n$.

The power indices characterized in Theorem 5.3 take the form

$$
\widetilde{\varphi}_{i}^{c}(C)=\sum_{j \in C(i) \backslash C(j)} \frac{\alpha_{\left|M_{j}^{C}\right|}}{\left|M_{j}^{C}\right|}-\alpha_{\left|M_{i}^{C}\right|} 1_{\left\{M_{i}^{C} \neq \emptyset, i \notin C(i)\right\}},
$$

with $\alpha_{k}$ as in Theorem 5.3. In control terms, according to a power index $\widetilde{\varphi}^{c}$, if player $j$ controls himself, then no player, including player $j$, derives (positive or negative) power from controlling $j$. Further, the (negative, if $c>0$ ) power from being controlled decreases and converges to zero as the number of controlling players increases. We note that $\widetilde{\varphi}^{c}$ is related to the apex power index in van den Brink (2002). 
Proof of Theorem 5.3 For the if-direction let $c \in \mathbb{R}$ and $\widetilde{\varphi}^{c}$ as in (5). We show that $\widetilde{\varphi}^{c}$ satisfies LA3. Let $C, C^{\prime}, j, i$ be as in the statement of the axiom, and $k=\left|M_{j}^{C}\right| \in\{1, \ldots, n-1\}$. First, if $j \notin M_{j}^{C}, i \neq j$, then

$$
\widetilde{\varphi}_{i}^{c}\left(C^{\prime}\right)-\widetilde{\varphi}_{i}^{c}(C)=\frac{\alpha_{k+1}}{k+1}=\frac{2 c}{(k+1)(k+2)}
$$

and

$$
\widetilde{\varphi}_{j}^{c}\left(C^{\prime}\right)-\widetilde{\varphi}_{j}^{c}(C)=-\alpha_{k+1}+\alpha_{k}=\frac{2 c}{(k+1)(k+2)}
$$

so that LA3 holds. Second, if $j \in M_{j}^{C}, i \neq j$, then

$$
\widetilde{\varphi}_{i}^{c}\left(C^{\prime}\right)-\widetilde{\varphi}_{i}^{c}(C)=\frac{\beta_{k+1}}{k+1}=0
$$

and

$$
\widetilde{\varphi}_{j}^{c}\left(C^{\prime}\right)-\widetilde{\varphi}_{j}^{c}(C)=\frac{\beta_{k+1}}{k+1}-\beta_{k+1}-\left(\frac{\beta_{k}}{k}-\beta_{k}\right)=0 .
$$

Also here LA3 holds.

For the only-if direction, let $\varphi=\varphi^{\alpha, \beta} \in \Phi$ satisfy LA3. First suppose $M \in$ $P_{0}(N)$ and $i, j \in N \backslash M$ with $i \neq j$ and $|M|=k$ for some $k \in\{1, \ldots, n-2\}$. Then $\varphi_{j}\left(U_{M \cup\{i\}, j}\right)-\varphi_{j}\left(U_{M, j}\right)=-\alpha_{k+1}+\alpha_{k}$, whereas $\varphi_{i}\left(U_{M \cup\{i\}, j}\right)-\varphi_{i}\left(U_{M, j}\right)=$ $\alpha_{k+1} /(k+1)$. By LA3 with $U_{M, j}$ in the role of $C$ and $U_{M \cup\{i\}, j}$ in the role of $C^{\prime}$, this implies $\alpha_{k}=\frac{2}{k+1} c$ for all $k=1, \ldots, n-1$ with $\alpha_{1}=c$.

Now suppose $M^{\prime} \in P_{0}(N)$ with $j \in M^{\prime}, i \notin M^{\prime}$, and $\left|M^{\prime}\right|=k \in\{1, \ldots, n-$ $1\}$. Then $\varphi_{j}\left(U_{M^{\prime} \cup\{i\}, j}\right)-\varphi_{j}\left(U_{M^{\prime}, j}\right)=-k \beta_{k+1} /(k+1)+(k-1) \beta_{k} / k$, and $\varphi_{i}\left(U_{M^{\prime} \cup\{i\}, j}\right)-\varphi_{i}\left(U_{M^{\prime}, j}\right)=\beta_{k+1} /(k+1)$. By LA3, this implies $\beta_{k+1}=((k-$ $1) / k) \beta_{k}$ for all $k \in\{1, \ldots, n-1\}$. Since $\beta_{1}=0$, it follows that $\beta_{k}=0$ for all $k=2, \ldots, n$.

Remark 5.4. If we weaken LA3 to a condition LA3' by strengthening the premiss that $M_{j}^{C} \neq \emptyset$ to $M_{j}^{C} \backslash\{j\} \neq \emptyset$ like in LA1 and LA2, then we obtain a two-parameter family: there is a $c \in \mathbb{R}$ such that $\alpha_{k}=\frac{2}{k+1} c$ for all $k=$ $1, \ldots, n-1$, and a $d \in \mathbb{R}$ such that $\beta_{k}=\frac{1}{k-1} d$ for all $k=2, \ldots, n$. We omit the proof of this claim.

\section{Concluding remarks}

We provide a summary and further relations with the literature, and finally show that the axioms in Theorem 4.4 are independent.

\subsection{Summary and further relations with the literature}

In this paper we axiomatized a class of power indices for invariant digraphs by the axioms of Constant Sum, Anonyimity, Null Player and the Transfer Property, inspired by Karos and Peters (2015), see Theorem 4.4. By adding 
three different link addition axioms we obtained three subclasses, which are related to the Copeland score, $\beta$-measure and apex measure. A main difference with the last two measures is that these satisfy a normalization where the total sum of the power of all players depends on the digraph, whereas in the indices of this paper, this sum is always zero. As a consequence, in the present paper players can have negative power, which is not possible in the $\beta$-measure and apex measure.

The $\beta$ - and apex measures also satisfy Anonymity and Null Player, and the $\beta$-measure even satisfies a stronger version of NP, in which every player who has no outgoing arcs has power zero. As we saw in this paper and as also follows from Karos and Peters (2015) in a more general model, the Transfer and Null Player properties imply that we can find the power values of a digraph by adding up the power values over special elementary digraphs, one associated with every player, where such a digraph consists of all arcs going into this player (see Lemma 4.2). For digraphs this is also implied by Additivity over Independent Partitions used in van den Brink and Gilles (2000) to axiomatize the outdegree and $\beta$ measure, and requiring that the power in a digraph is the sum of the powers in a partition of the digraph such that every player has a positive indegree in at most one subgraph in the partition.

The Copeland score for digraphs assigns to every player the difference between its outdegree and indegree. Therefore, the power indices satisfying LA1 characterized in Theorem 5.1 can be seen as multiples of the Copeland score.

The power indices characterized by adding LA2 (see Theorem 5.2) are similar to multiples of the $\beta$-measure ${ }^{9}$, with the exception that players who are dominated but do not dominate have a negative power, while they have a zero power in the $\beta$-measure (and even positive power in the reflexive $\beta$-measure). This fits well with the interpretation of mutual control.

The power indices characterized by adding LA3 (see Theorem 5.3) are similar to multiples of the apex measure, with the exception that players who are dominated but do not dominate have negative power, while they have positive power in the apex-measure. Similar to the apex measure, according to these indices, a player who is dominated gets higher power if he gets dominated by more other predecessors. This reflects that the control over a player decreases when more other players are going to control this player, and therefore its 'self-control' increases, but will never become positive.

We illustrate the different classes of power indices we considered here by an example.

Example 6.1. For $N=\{1, \ldots, n\}$ and $k \in N \backslash\{n\}$, consider the structures $C_{n}^{k} \in \mathcal{D}^{*}$ given by $C_{n}^{k}(i)=\{n\}$ for all $i \in\{1, \ldots, k\}$, and $C_{n}^{k}(i)=\emptyset$ otherwise. In Table 1 we give the powers of successor $n$ and predecessors $i \in M_{n}^{C}$ for different numbers of predecessors.

Finally, we remark that for an arbitrary digraph $C \in \mathcal{D}$ one could define $\varphi(C)=\varphi\left(C^{*}\right)$ where $C^{*}$ is the invariant extension of $C$ obtained as explained

\footnotetext{
${ }^{9}$ The $\beta$-measure for (irreflexive) digraphs assigns to every player the sum over all its successors of 1 divided by the number of predecessors of this successor.
} 


\begin{tabular}{|c|c|c|c|c|c|c|}
\hline & 1 & 2 & 3 & 4 & $\ldots$ & $k$ \\
\hline \hline$\varphi_{i}^{c}, i \in M_{n}^{C}$ & $c$ & $c$ & $c$ & $c$ & $\ldots$ & $c$ \\
\hline$\varphi_{n}^{c}$ & $-c$ & $-2 c$ & $-3 c$ & $-4 c$ & $\ldots$ & $-k c$ \\
\hline \hline $\bar{\varphi}_{i}^{c}, i \in M_{n}^{C}$ & $c$ & $\frac{c}{2}$ & $\frac{c}{3}$ & $\frac{c}{4}$ & $\ldots$ & $\frac{c}{k}$ \\
\hline $\bar{\varphi}_{n}^{c}$ & $-c$ & $-c$ & $-c$ & $-c$ & $\ldots$ & $-c$ \\
\hline \hline$\widetilde{\varphi}_{i}^{c}, i \in M_{n}^{C}$ & $c$ & $\frac{c}{3}$ & $\frac{c}{6}$ & $\frac{c}{10}$ & $\ldots$ & $\frac{2 c}{k(k+1)}$ \\
\hline$\widetilde{\varphi}_{n}^{c}$ & $-c$ & $-\frac{2 c}{3}$ & $-\frac{c}{2}$ & $-\frac{2 c}{5}$ & $\ldots$ & $-\frac{2 c}{k+1}$ \\
\hline
\end{tabular}

Table 1: Power indices of Example 6.1

in Section 2, namely by adding arcs for every pair of players between whom there exists a directed path.

\subsection{Independence of the axioms in Theorem 4.4}

By providing four examples we show that the axioms in the main characterization, Theorem 4.4, are logically independent.

Not NP but CS, AN, TP For $U_{M, j} \in \mathcal{D}^{*}$ with $M=\{j\}$ define $\varphi_{i}\left(U_{M, j}\right)=$ $1 /(n-1)$ for all $i \in N \backslash\{j\}$ and $\varphi_{j}\left(U_{M, j}\right)=-1$. In all other cases define $\varphi\left(U_{M, j}\right)$ as in Lemma 4.3. Extend to all $\mathcal{D}^{*}$ by taking sums as in Theorem 4.4 .

Not CS but NP, AN, TP For $U_{M, j} \in \mathcal{D}^{*}$ with $M=\{j\}$ define $\varphi_{i}\left(U_{M, j}\right)=0$ for all $i \in N \backslash\{j\}$ and $\varphi_{j}\left(U_{M, j}\right)=1$. In all other cases define $\varphi\left(U_{M, j}\right)$ as in Lemma 4.3. Extend to all $\mathcal{D}^{*}$ by taking sums as in Theorem 4.4 .

Not AN but NP, CS, TP For $U_{M, j} \in \mathcal{D}^{*}$ with $|M|=2$ and $j \notin M$ (hence $n \geq 3)$ define $\varphi_{i}\left(U_{M, j}\right)=0$ for all $i \notin M \cup\{j\}$ also for $i \in M$ when $i>k$ and $M=\{i, k\}, \varphi_{k}\left(U_{M, j}\right)=1$, and $\varphi_{j}\left(U_{M, j}\right)=-1$. In all other cases define $\varphi\left(U_{M, j}\right)$ as in Lemma 4.3. Extend to all $\mathcal{D}^{*}$ by taking sums as in Theorem 4.4 .

Not TP but NP, CS, AN For $C \in \mathcal{D}^{*}$ define

$n_{1}=\left|\left\{i \in N: C(i) \neq \emptyset, C_{i}=O\right\}\right|, n_{2}=\left|\left\{i \in N: C(i)=\emptyset, C_{i} \neq O\right\}\right|$.

If $n_{1}, n_{2}>0$ then define

$$
\varphi_{i}(C)=\left\{\begin{array}{cl}
1 / n_{1} & \text { if } C(i) \neq \emptyset, C_{i}=O \\
-1 / n_{2} & \text { if } C(i)=\emptyset, C_{i} \neq O \\
0 & \text { otherwise }
\end{array}\right.
$$

and define $\varphi(C)=(0, \ldots, 0)$ in all other cases. To see that $\varphi$ does not satisfy $\mathrm{TP}$, let $n=4$ and consider $C, D \in \mathcal{D}^{*}$ with $C(1)=2$, $D(3)=4$, and $C(S), D(S)=\emptyset$ in all other cases. Then $\varphi(C \cup D)+$ $\varphi(C \cap D)=(1 / 2,-1 / 2,1 / 2,-1 / 2)$ whereas $\varphi(C)=(1,-1,0,0)$ and $\varphi(D)=(0,0,1,-1)$, thus violating $(1)$ and therefore TP. 


\section{References}

Copeland AH (1951) A 'reasonable' social welfare function. University of Michigan Seminar on Applications of Mathematics to the Social Sciences

Dubey P (1975) On the uniqueness of the Shapley value. International Journal of Game Theory 4:131-139

Dubey P, Einy E, Haimanko O (2005) Compound voting and the Banzhaf index. Games and Economic Behavior 51:20-30

Einy E, Haimanko O (2011) Characterization of the Shapley-Shubik power index without the efficiency axiom. Games and Economic Behavior 73:615621

Gambarelli G (1994) Power indices for political and financial decision making. Annals of Operations Research 51:163-173

Gambarelli G, Owen G (1994) Indirect control of corporations. International Journal of Game Theory 23:287-302

Gambarelli G, Stach I (2009) Power indices in politics: some results and open problems. Homo Oeconomics 26:417-441

Gilles RP, Owen G, van den Brink R (1992) Games with permission structures: the conjunctive approach. International Journal of Game Theory 20:277293

Hu X, Shapley LS (2003a) On authority distributions in organizations: equilibrium. Games and Economic Behavior 45:132-152

Hu X, Shapley LS (2003b) On authority distributions in organizations: controls. Games and Economic Behavior 45:153-170

Karos D, Peters H (2015) Indirect control and power in mutual control structures. Games and Economic Behavior 92:150-165

Myerson RB (1977) Graphs and Cooperation in Games. Mathematics of Operations Research 2: 225-229.

Shapley LS (1953) A value for $n$-person games. In: Kuhn HW, Tucker AW (eds) Contributions to the Theory of Games II, Princeton University Press, Princeton.

Shapley LS, Shubik M (1954) A method for evaluating the distribution of power in a committee system. American Political Science Review 48:787-792

van den Brink R (2000) The Apex Power Measure for Directed Networks. Social Choice and Welfare 19: 845-867

van den Brink R, Borm P (2002) Digraph Competitions and Cooperative Games. Theory and Decision 53: 327-342. 
van den Brink R, Gilles R (2000) Measuring domination in directed networks.

Social Networks 22:141-157 